Communications in Physics, Vol.23, No. 1 (2013), pp. 11-19

\title{
WARD-TAKAHASHI IDENTITY FOR VERTEX FUNCTIONS OF SQED
}

\author{
HA THANH HUNG \\ Department of Physics, Hanoi University of Education No. 2 \\ LE THO HUE AND HOANG NGOC LONG \\ Institute of Physics, VAST
}

\begin{abstract}
Ward-Takahashi identity is an useful tool for calculating amplitude of scattering processes. In the high-order perturbative theory of $s Q E D$, propagator and vertex functions contain many high-order corrections. By using Ward-Takahashi identity, each vertex function is separated into two parts: "longitudinal" and "transverse "part. The longitudinal part can be directly calculated from Ward-Takahashi identity. The transverse part depends on the expanding of specific orders of the theory. In this report, we present one method based on the Ward-Takahashi identity, to calculate this part of vertex functions at the one-loop order in arbitrary gauge and dimensions in $s Q E D$.
\end{abstract}

\section{INTRODUCTION}

We introduce a method in which Ward-Takahashi identity is used to decompose the vertex into to longitudinal part and transverse part. This form of vertex satisfies two conditions: (i) has no kinematics singularities in both two parts, (ii) the longitudinal part of a vertex has been fixed by scalar coefficient.

\section{PROPAGATORS AND VERTEX FUNCTIONS OF sQED IN BARE PERTURBATION.}

In the scalar Quantum Electrodynamics Dynamics (sQED), propagator and vertex functions in any gauge $\xi$ are determined as follows [1]:

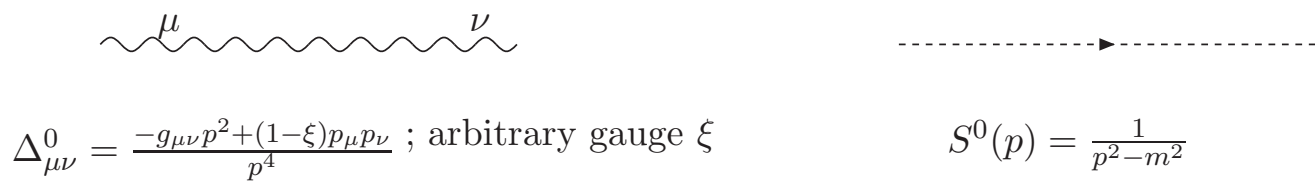

Fig. 1. Propagators of sQED in bare perturbative theory 


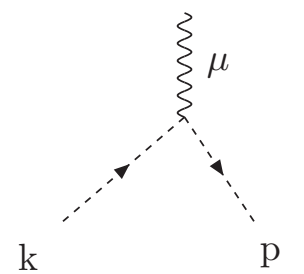

$\Gamma_{\mu}^{0}=(k+p)^{\mu}$

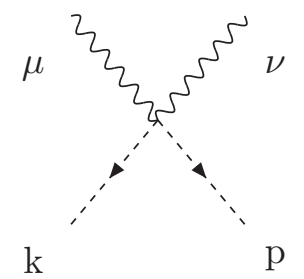

$e^{2} \Gamma_{\mu \nu}^{0}=e^{2} g_{\mu \nu}$

Fig. 2. Vertex functions of SQED in bare perturbative theory

\section{WARD-TAKAHASHI IDENTITY WITH 3-POINT VERTEX FUNCTION OF sQED .}

Propagators of scalar particles at one-loop order [1]:

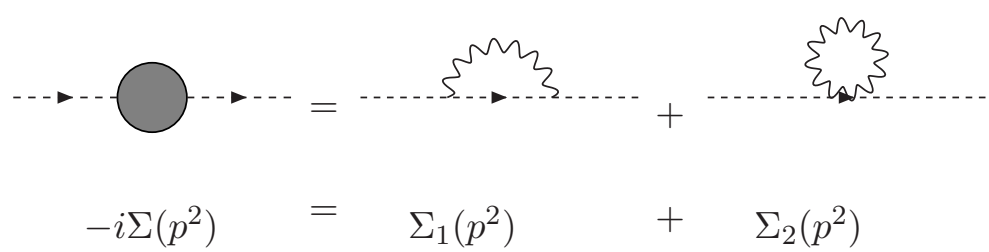

Fig. 3. Propagator of complex scalar particle at one-loop.

In dimensional regularization, the second diagram (tadpole) vanishes, thus only first diagram contribute $[2,3]$ The one-loop propagator is given by:

$$
\begin{aligned}
S^{-1} & =\frac{-e^{2}}{m^{2}}\left(\frac{m^{2}}{4 \pi}\right)^{l} \Gamma(1-l)\left\{1-\frac{2\left(m^{2}+p^{2}\right)}{m^{2}} F_{2}\left(2-l, 1 ; l ; \frac{p^{2}}{m^{2}}\right)\right. \\
& \left.\left.+(1-\xi) \frac{\left(m^{2}-p^{2}\right)^{2}}{m^{4}}\right)_{2} F_{1}\left(3-l, 2 ; l ; \frac{p^{2}}{m^{2}}\right)\right\}
\end{aligned}
$$

The 3-point function of sQED at one-loop is illustrated by following diagrams[2]:

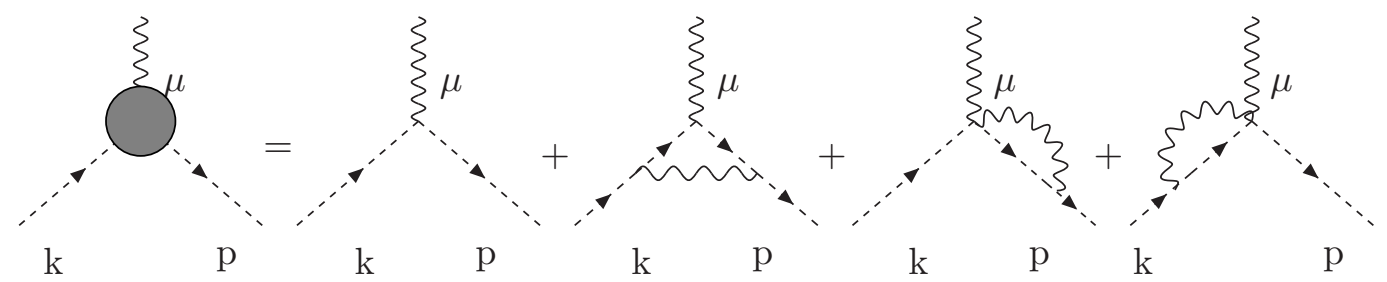

In terms of mathematical language, we have

$$
\Gamma^{\mu}(k, p)=(k+p)^{\mu}+\Gamma_{1}^{\mu}(k, p)+\Gamma_{2}^{\mu}(p)+\Gamma_{2}^{\mu}(k)
$$

in which: 

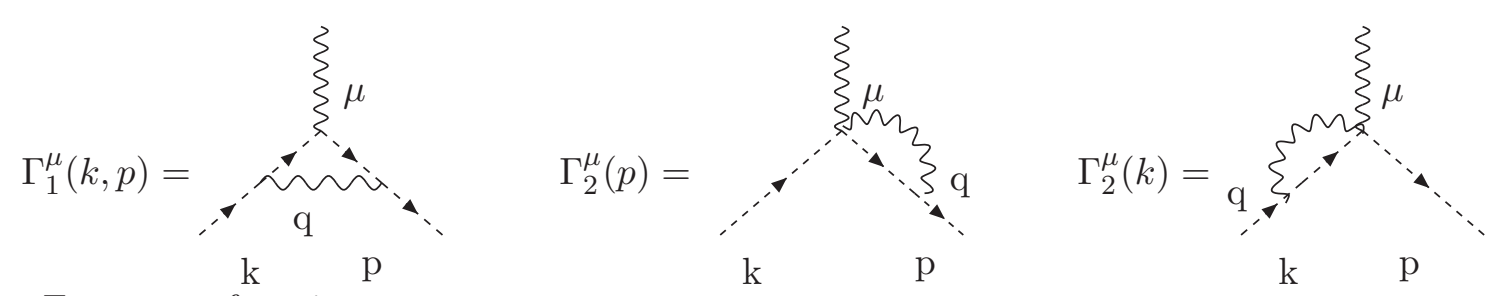

For vertex functions:

$$
\begin{aligned}
\Gamma_{\mu}^{1} & =\frac{-i e^{2}}{(2 \pi)^{2 l}}\left\{4(k p)(k+p)_{\mu} J^{0}+\left[-8(k p) g_{\mu}^{\nu}-2(k+p)_{\mu}(k+p)^{\nu}\right] J_{\nu}^{1}+4(k+p)^{\nu} J_{\mu \nu}^{2}\right. \\
& +(k+p)_{\mu} K^{0}-2 K_{\mu}^{1}+(\xi-1)\left[(k+p)_{\mu} K^{0}+4(k+p)_{\mu} P^{\alpha} K^{\beta} I_{\alpha \beta}^{2}-8 p^{\alpha} K^{\beta} I_{\mu \alpha \beta}^{3}\right. \\
& \left.\left.-2(k+p)_{\mu}(k+p)^{\alpha} J_{\alpha}^{1}+4(k+p)^{\alpha} J_{\mu \alpha}^{2}-2 K_{\mu}^{1}\right]\right\}
\end{aligned}
$$

and

$$
\begin{aligned}
\Gamma_{2}^{\mu}(p) & =\frac{e^{2} p^{2} p^{\mu}}{(2 \pi)^{2 l}}\left\{\left[3+\frac{m^{2}}{p^{2}}\right] Q_{1}(p)-\frac{\pi^{l-2}}{p^{2}} \Gamma(1-l)\left(m^{2}\right)^{l-1}\right. \\
& \left.+(\xi-1) \frac{p^{2}-m^{2}}{p^{2}}\left[Q_{1}(p)+\left(p^{2}-m^{2}\right) Q_{3}(p)\right]\right\}
\end{aligned}
$$

The Ward-Takahashi identity for the 3-point vertex function reads [4]:

$$
q_{\mu} \Gamma^{\mu}(k, p)=S^{-1}(k)-S^{-1}(p)
$$

in higher corrective orders we introduce:

$$
\Gamma^{\mu}(k, p)=\Gamma_{L}^{\mu}(k, p)+\Gamma_{T}^{\mu}(k, p)
$$

The longitudinal component and the transverse component are obeyed as functions depend on $k, p, q[2]$.

$$
\Gamma_{L}^{\mu}(k, p)=\frac{S^{-1}(k)-S^{-1}(p)}{k^{2}-p^{2}}(k+p)^{\mu} ; \Gamma_{T}^{\mu}(k, p)=\tau\left(k^{2}, p^{2}, q^{2}\right) T^{\mu}(k, p)
$$

where

$$
T^{\mu}(k, p)=p q k^{\mu}-k q p^{\mu}=\frac{1}{2}\left[q^{\mu}\left(k^{2}-p^{2}\right)-(k+p)^{\mu} q^{2}\right]
$$

The condition for $\Gamma_{T}^{\mu}(k, p)$ is:

$$
q_{\mu} \Gamma_{T}^{\mu}(k, p)=0 ; \Gamma_{T}^{\mu}(p, p)=0
$$


The function $\tau\left(k^{2}, p^{2}, q^{2}\right)$ is reduced as follows:

$$
\begin{aligned}
\tau\left(k^{2}, p^{2}, q^{2}\right) & =\frac{e^{2} \pi^{2}}{2(2 \pi)^{d} \Delta^{2}}\left\{\left(k^{2}-2 m^{2}+p^{2}-4 k p\right)\left[-K_{0}+\left(m^{2}+k p\right) J_{0}\right]\right. \\
& +\frac{2 Q_{1}(p)}{k^{2}-p^{2}}\left[p^{2}\left(p^{2}-3 k p\right)+k^{2}\left(k p-3 p^{2}\right)-2 m^{2}\left(p^{2}+k p\right)\right] \\
& -\frac{2 Q_{1}(k)}{k^{2}-p^{2}}\left[k^{2}\left(k^{2}-3 k p\right)+p^{2}\left(k p-3 k^{2}\right)-2 m^{2}\left(k^{2}+k p\right)\right] \\
& +(\xi-1)\left(m^{2}-k^{2}\right)\left(m^{2}-p^{2}\right)\left[J_{0}-\left(k p+m^{2}\right) I_{0}-\frac{2 Q_{3}(p)}{k^{2}-p^{2}}\left(k p+p^{2}\right)\right. \\
& \left.\left.-\frac{2 Q_{3}(k)}{k^{2}-p^{2}}\left(k p+k^{2}\right)\right]\right\}
\end{aligned}
$$

in which:

$$
\Delta^{2}=(k p)^{2}-k^{2} p^{2}=(k q)^{2}-k^{2} q^{2}
$$

It is convenient to present $\tau\left(k^{2}, p^{2}, q^{2}\right)$ in terms of propagators of scalar particle:

$$
\begin{aligned}
\tau\left(k^{2}, p^{2}, q^{2}\right)= & \frac{1}{4 \Delta^{2}} \frac{\left[S^{-1}(k, \xi=1)-S^{-1}(p, \xi=1)\right]}{\left[\left(m^{2}+k^{2}\right) Q_{1}(k)-\left(m^{2}+p^{2}\right) Q_{1}(p)\right]}\left\{\left(k^{2}-2 m^{2}+p^{2}-4 k p\right)\right. \\
& \times\left[-K_{0}+\left(m^{2}+k p\right) J_{0}\right] \\
+ & \frac{2 Q_{1}(p)}{k^{2}-p^{2}}\left[p^{2}\left(p^{2}-3 k p\right)+k^{2}\left(k p-3 p^{2}\right)-2 m^{2}\left(p^{2}+k p\right)\right] \\
- & \left.\frac{2 Q_{1}(k)}{k^{2}-p^{2}}\left[k^{2}\left(k^{2}-3 k p\right)+p^{2}\left(k p-3 k^{2}\right)-2 m^{2}\left(k^{2}+k p\right)\right]\right\} \\
+ & \frac{1}{2 \Delta^{2}} \frac{\left[S^{-1}(k, \xi-1)-S^{-1}(p, \xi-1)\right]}{\left[\left(m^{2}-k^{2}\right) Q_{3}(k)-\left(m^{2}-p^{2}\right) Q_{3}(p)\right]} \\
\times & \left(m^{2}-k^{2}\right)\left(m^{2}-p^{2}\right)\left\{J_{0}-\left(k p+m^{2}\right) I_{0}\right. \\
- & \left.\frac{2 Q_{3}(p)}{k^{2}-p^{2}}\left(k p+p^{2}\right)-\frac{2 Q_{3}(k)}{k^{2}-p^{2}}\left(k p+k^{2}\right)\right\} .
\end{aligned}
$$

We define

$$
q^{\mu}=(k-p)^{\mu} ; P^{\mu}=(k+p)^{\mu}
$$

then Ward-Takahashi identity for three-point function can be written in the form

$$
\begin{aligned}
q_{\mu} \Gamma_{\nu}-q_{\nu} \Gamma_{\mu} & =\left(q_{\mu} P_{\nu}-q_{\nu} P_{\mu}\right)\left[\frac{S^{-1}(k)-S^{-1}(p)}{k^{2}-p^{2}}+\frac{q^{2}}{2} \tau\left(k^{2}, p^{2}, q^{2}\right)\right], \\
P_{\mu} \Gamma_{\nu}-P_{\nu} \Gamma_{\mu} & =\left(P_{\mu} q_{\nu}-P_{\nu} q_{\mu}\right)\left[\frac{k^{2}-p^{2}}{2}\right] \tau\left(k^{2}, p^{2}, q^{2}\right)
\end{aligned}
$$




\section{WARD-TAKAHASHI IDENTITY WITH 4-POINT VERTEX FUNCTION OF sQED.} by:

The Ward-Takahashi identity of 4-point function relates to 3-point vertex function

$$
\begin{aligned}
k^{\prime \mu} \Gamma_{\nu \mu}\left(p^{\prime}, k^{\prime} ; p, k\right) & =\Gamma_{\nu}(p+k, p)-\Gamma_{\nu}\left(p^{\prime}, p^{\prime}-k\right), \\
k^{\mu} \Gamma_{\nu \mu}\left(p^{\prime}, k^{\prime} ; p, k\right) & =\Gamma_{\nu}\left(p^{\prime}, p^{\prime}+k^{\prime}\right)-\Gamma_{\nu}\left(p-k^{\prime}, p\right)
\end{aligned}
$$

Following Eq. (13) we can determine the longitudinal component of 4-point vertex function based on 3-point vertex functions[3]. Let us denote:

$$
\begin{gathered}
Q_{\mu}=k_{\mu}^{\prime}\left(p+p^{\prime}\right) k-k k^{\prime}\left(p+p^{\prime}\right)_{\mu} ; R_{\mu}=k_{\mu} k^{\prime} k-k^{\prime 2} k_{\nu}, \\
Q_{\nu}^{\prime}=k_{\nu}\left(p+p^{\prime}\right) k^{\prime}-k k^{\prime}\left(p+p^{\prime}\right)_{\nu} ; R_{\nu}^{\prime}=k_{\nu}^{\prime} k^{\prime} k-k^{\prime 2} k_{\nu}
\end{gathered}
$$

then 4 -point vertex function is written in the form:

$$
\begin{aligned}
\Gamma_{\mu \nu} & =\Gamma_{\mu \nu}^{L}+\Gamma_{\mu \nu}^{T}=A g_{\mu \nu}+B_{11}\left(k k^{\prime} g_{\mu \nu}-k_{\nu} k_{\mu}^{\prime}\right)+B_{12} Q_{\nu}^{\prime} k_{\mu}^{\prime}+B_{13} R_{\nu}^{\prime} k_{\mu}^{\prime} \\
& +B_{21} k_{\nu} Q_{\mu}+B_{22} Q_{\nu}^{\prime} Q_{\mu}+B_{23} R_{\nu}^{\prime} Q_{\mu}+B_{31} k_{\nu} R_{\mu}+B_{32} Q_{\nu}^{\prime} R_{\mu}+B_{33} R_{\nu}^{\prime} R_{\mu}
\end{aligned}
$$

Now we can determine longitudinal and transverse components of 4-point vertex function as follows:

$$
\begin{aligned}
& \Gamma_{\mu \nu}^{L}=A g_{\mu \nu}+B_{12} Q_{\nu}^{\prime} k_{\mu}^{\prime}+B_{13} R_{\nu}^{\prime} k_{\mu}^{\prime}+B_{21} k_{\nu} Q_{\mu}+B_{31} k_{\nu} R_{\mu} \\
& \Gamma_{\mu \nu}^{T}=B_{11}\left(k k^{\prime} g_{\mu \nu}-k_{\nu} k_{\mu}^{\prime}\right)+B_{22} Q_{\nu}^{\prime} Q_{\mu}+B_{23} R_{\nu}^{\prime} Q_{\mu}+B_{32} Q_{\nu}^{\prime} R_{\mu}+B_{33} R_{\nu}^{\prime} R_{\mu}
\end{aligned}
$$

Factors of longitudinal component of 4 -point vertex function is given by:

$$
\begin{aligned}
A & =-\frac{1}{k k^{\prime}}\left\{S^{-1}(p+k)-S^{-1}(p)+S^{-1}\left(p^{\prime}-k\right)-S^{-1}\left(p^{\prime}\right)\right\}, \\
B_{21} & =-\frac{1}{k k^{\prime}}\left\{\frac{S^{-1}(p+k)-S^{-1}(p)}{(p+k)^{2}-p^{2}}-\frac{S^{-1}\left(p^{\prime}\right)-S^{-1}\left(p^{\prime}-k\right)}{p^{\prime 2}-\left(p^{\prime}-k\right)^{2}}\right. \\
& \left.-k^{2}\left[\Gamma_{T}(p+k, p)-\Gamma_{T}\left(p^{\prime}, p^{\prime}-k\right)\right]\right\} \\
B_{12} & =-\frac{1}{k k^{\prime}}\left\{\frac{S^{-1}\left(p^{\prime}-k^{\prime}\right)-S^{-1}\left(p^{\prime}\right)}{\left(p^{\prime}-k^{\prime}\right)^{2}-p^{\prime 2}}-\frac{S^{-1}(p)-S^{-1}\left(p+k^{\prime}\right)}{p^{2}-\left(p+k^{\prime}\right)^{2}}\right. \\
& \left.-k^{\prime 2}\left[\Gamma_{T}\left(p^{\prime}-k^{\prime}, p^{\prime}\right)-\Gamma_{T}\left(p, p+k^{\prime}\right)\right]\right\}, \\
B_{31} & =-\frac{1}{\left(k k^{\prime}\right)^{2}}\left\{\left[(p+k)^{2}-p^{2}\right] \Gamma_{T}(p+k, p)+\left[\left(p^{\prime}-k\right)^{2}-p^{\prime 2}\right] \Gamma_{T}\left(p^{\prime}, p^{\prime}-k\right)\right\}, \\
B_{13} & =-\frac{1}{\left(k k^{\prime}\right)^{2}}\left\{\left[\left(p^{\prime}-k^{\prime}\right)^{2}-p^{\prime 2}\right] \Gamma_{T}\left(p^{\prime}-k^{\prime}, p^{\prime}\right)+\left[\left(p+k^{\prime}\right)^{2}-p^{2}\right] \Gamma_{T}\left(p, p-+k^{\prime}\right)\right\}
\end{aligned}
$$

in which $\Gamma_{T}$ is the transverse component of 3-point vertex function and determined as follows:

$$
\Gamma_{\mu}(p+k, p)=(2 p+k)_{\mu} \frac{S^{-1}(p+k)-S^{-1}(p)}{(p+k)^{2}-p^{2}}+2\left(k_{\mu} p k-k^{2} p_{\mu}\right) \Gamma_{T}(p+k, p)
$$

Factors $B_{11}, B_{22}, B_{23}, B_{32}$ and $B_{33}$ of transverse component of 4-point vertex function will be calculated according to respective order of perturbative theory. We will introduce 
technique to calculate the transverse component of 4-point vertex function at one-loop level. The corresponding Feynman diagrams for this function are given in Fig. 4 [3].

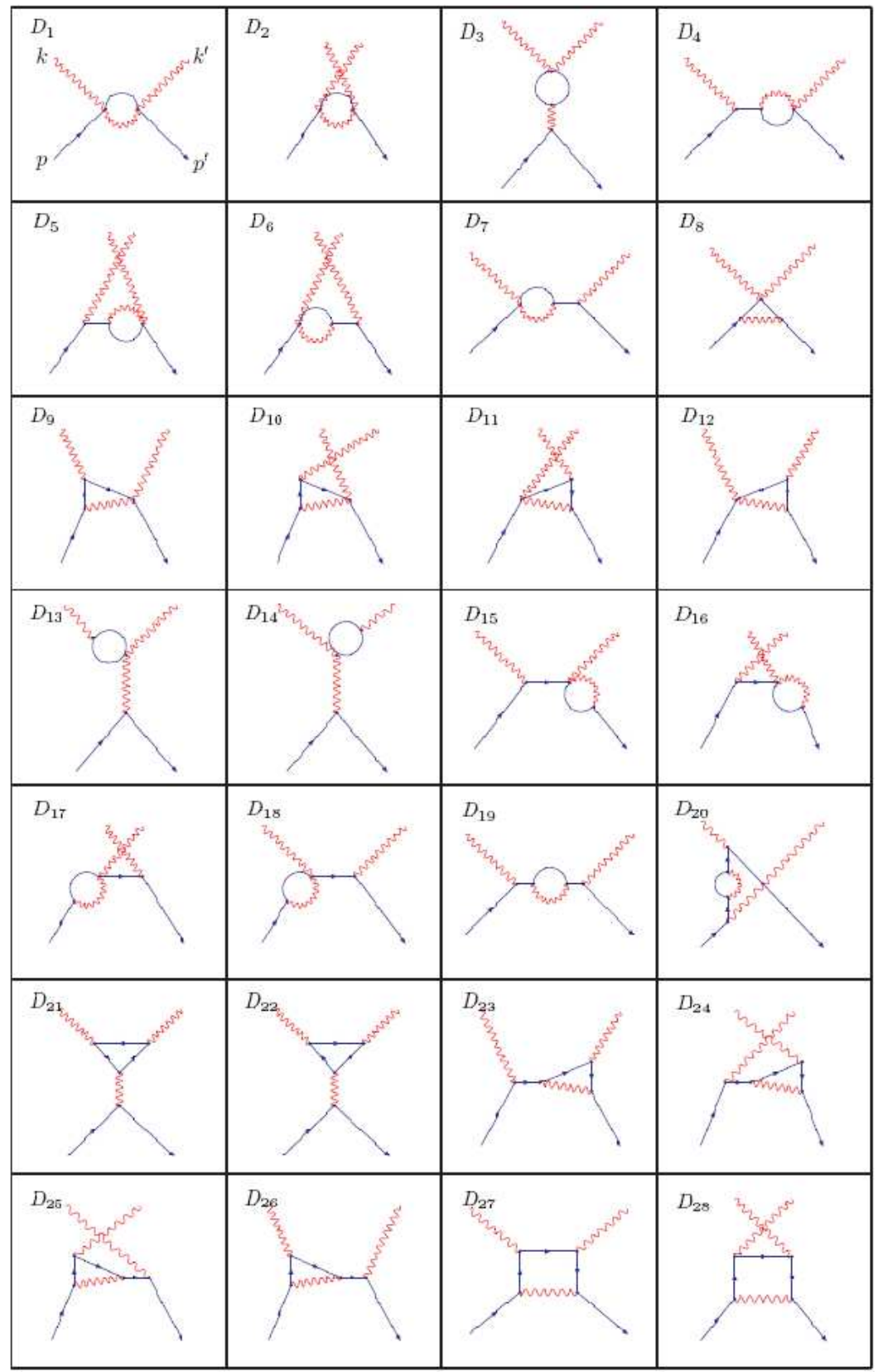

Fig. 4. Diagrams of 4-point vertex function 
For the sake of simplicity, we denote $q=k+p$ and $q^{\prime}=k^{\prime}+p$, then results of Feynman diagrams at one-loop are given by

$$
\begin{aligned}
& \Gamma_{D_{1}}^{\mu \nu}(q)=-\frac{2 i e^{2}}{(2 \pi)^{D}}\left\{-g^{\mu \nu} K(q)+(1-\xi) L^{\mu \nu}(q)\right\}, \\
& \Gamma_{D_{4}}^{\mu \nu}(p, q)=\frac{i e^{2}}{(2 \pi)^{D}}\left\{-2 \frac{(p+q)^{\mu} q^{\nu}}{q^{2}-m^{2}} K(q)+\frac{(p+q)^{\mu}}{q^{2}-m^{2}} K^{\nu}(q)+(1-\xi)(p+q)^{\mu} L^{\nu}(q)\right\}, \\
& \Gamma_{D_{8}}^{\mu \nu}(p, q)=-\frac{i e^{2}}{(2 \pi)^{D}} g^{\mu \nu}\left\{\tilde{K}\left(p-p^{\prime}\right)-2\left(p+p^{\prime}\right)^{\mu} I_{\mu}\left(p, p^{\prime}\right)+4 p p^{\prime} I\left(p, p^{\prime}\right)\right. \\
& \left.+(\xi-1) \tilde{K}\left(p-p^{\prime}\right)-2\left(p+p^{\prime}\right)^{\mu} I_{\mu}\left(p \cdot p^{\prime}\right)+4 p^{\prime \mu} p^{\nu} J_{\mu \nu}\left(p, p^{\prime}\right)\right\}, \\
& \Gamma_{D_{9}}^{\mu \nu}(p, q)=-\frac{i e^{2}}{(2 \pi)^{D}}\left\{2(p+q)^{\mu} p^{\nu} I(p, q)-(p+q)^{\mu} I^{\nu}(p, q)-4 p^{\nu} I^{\mu}(p+q)\right. \\
& +2 I^{\mu \nu}(p, q)-(1-\xi)\left[\left(p^{2}-m^{2}\right)(p+q)^{\mu}\right] J^{\nu}(p, q)-2\left(p^{2}-m^{2}\right) J^{\mu \nu}(p, q) \\
& \left.+2 L^{\mu \nu}(q)-(p+q)^{\mu} L^{\nu}(q)\right\} \text {, } \\
& \Gamma_{D_{15}}^{\mu \nu}\left(p, q, p^{\prime}\right)=\frac{i e^{2}}{(2 \pi)^{D}}\left\{-2 p^{\prime \nu} K\left(p^{\prime}\right)+K^{\nu}\left(p^{\prime}\right)+(1-\xi)\left(p^{\prime 2}-m^{2}\right) L^{\nu}\left(p^{\prime}\right)\right\}, \\
& \Gamma_{D_{19}}^{\mu \nu}\left(p, q, p^{\prime}\right)=\frac{i e^{2}}{2(2 \pi)^{D}}(p+q)^{\mu}\left(p^{\prime}+q\right)^{\nu}\left\{\left(\frac{2}{q^{2}-m^{2}}+\frac{4 m^{2}}{\left(q^{2}-m^{2}\right)^{2}}\right) K(q)\right. \\
& \left.-\frac{T}{\left(q^{2}-m^{2}\right)^{2}}-\frac{(1-\xi)}{q^{2}-m^{2}} L(q)\right\} \text {, } \\
& \Gamma_{D_{20}}^{\mu \nu}\left(p, q, p^{\prime}\right)=-\frac{i e^{2}}{2(2 \pi)^{D}} \frac{\left(p^{\prime}+q^{\prime}\right)^{\mu}\left(p+q^{\prime}\right)^{\nu}}{\left(q^{\prime 2}-m^{2}\right)^{2}}\left\{-4 q^{\prime 2} K\left(q^{\prime}\right)-T+4 q_{\alpha}^{\prime} K^{\alpha}\left(q^{\prime}\right)\right. \\
& \left.+(1-\xi)\left[T-4 q_{\alpha}^{\prime} K^{\alpha}\left(q^{\prime}\right)+4 q_{\alpha}^{\prime} q_{\beta}^{\prime} L^{\alpha \beta}\left(q^{\prime}\right)\right]\right\}
\end{aligned}
$$




$$
\begin{aligned}
\Gamma_{D_{23}}^{\mu \nu}\left(p, q, p^{\prime}\right) & =\frac{i e^{2}}{2(2 \pi)^{D}}\left\{( p + q ) ^ { \mu } ( p ^ { \prime } + q ) ^ { \nu } \left[\left(\frac{4 p^{\prime} q}{q^{2}-m^{2}}+\frac{m^{2}-p^{\prime 2}}{q^{2}-m^{2}}-1\right) I\left(p^{\prime}, q\right)\right.\right. \\
& \left.-\frac{\tilde{K}\left(p^{\prime}-q\right)}{q^{2}-m^{2}}+\frac{K\left(p^{\prime}\right)}{q^{2}-m^{2}}+\frac{K(q)}{q^{2}-m^{2}}\right] \\
& -2(p+q)^{\mu}\left[\left(\frac{4 p^{\prime} q}{q^{2}-m^{2}}+\frac{m^{2}-p^{\prime 2}}{q^{2}-m^{2}}-1\right) \times I^{\nu}\left(p^{\prime}, q\right)\right. \\
& \left.-\frac{\left(p^{\prime}+q\right)^{\nu}}{2\left(q^{2}-m^{2}\right)} \tilde{K}\left(p^{\prime}-q\right)+\frac{K^{\nu}\left(p^{\prime}\right)}{q^{2}-m^{2}}+\frac{K^{\nu}(q)}{q^{2}-m^{2}}\right] \\
& -(1-\xi)\left[(p+q)^{\mu}\left(p^{\prime}+q\right)^{\nu}\left[\left(p^{2}-m^{2}\right) J\left(p^{\prime}, q\right)-L(q)-\frac{p^{2}-m^{2}}{q^{2}-m^{2}} L\left(p^{\prime}\right)\right]\right. \\
& \left.\left.-2(p+q)^{\mu}\left[\left(p^{\prime 2}-m^{2}\right) J^{\nu}\left(p^{\prime}, q\right)-L^{\nu}(q)-\frac{p^{2}-m^{2}}{q^{2}-m^{2}} L^{\nu}\left(p^{\prime}\right)\right]\right]\right\} \\
& \\
\Gamma_{D_{27}}^{\mu \nu}\left(p, q, p^{\prime}\right) & =\frac{i e^{2}}{2(2 \pi)^{D}}\left\{( k + 2 p ) ^ { \mu } ( k + 6 p + p ^ { \prime } ) ^ { \nu } \left[\left(-2 m^{2}+\left(p^{\prime}-p\right)^{2}-2 p p^{\prime}\right) U\left(p \cdot p^{\prime} . q\right)\right.\right. \\
& \left.+\tilde{I}\left(q-p, p^{\prime}-p\right)-I(p, q)-I\left(p^{\prime}, q\right)\right]-2\left(k+p+p^{\prime \nu}\left[\left(-2 m^{2}+\left(p^{\prime}-p\right)^{2}\right.\right.\right. \\
& \left.\left.\left.\left.-2 p p^{\prime}\right) U^{\mu}\left(p, p^{\prime}, q\right)\right]\right)+\tilde{I}^{\mu}\left(q-p, p^{\prime}-p\right)\right\}
\end{aligned}
$$

The remain Feynman diagrams are determined according to:

$$
\begin{gathered}
\Gamma_{D_{2}}^{\mu \nu}=\Gamma_{D_{1}}^{\mu \nu}\left(q^{\prime}\right) ; \Gamma_{D_{5}}^{\mu \nu}=\Gamma_{D_{4}}^{\mu \nu}\left(p, q^{\prime}\right) ; \Gamma_{D_{6}}^{\mu \nu}=\Gamma_{D_{4}}^{\mu \nu}\left(p^{\prime}, q^{\prime}\right) ; \Gamma_{D_{7}}^{\mu \nu}=\Gamma_{D_{4}}^{\mu \nu}\left(p^{\prime}, q\right) \\
\Gamma_{D_{10}}^{\mu \nu}=\Gamma_{D_{9}}^{\mu \nu}\left(p, q^{\prime}\right) ; \Gamma_{D_{11}}^{\mu \nu}=\Gamma_{D_{9}}^{\mu \nu}\left(p^{\prime}, q^{\prime}\right) ; \Gamma_{D_{12}}^{\mu \nu}=\Gamma_{D_{9}}^{\mu \nu}\left(p^{\prime}, q\right) ; \Gamma_{D_{16}}^{\mu \nu}=\Gamma_{D_{15}}^{\mu \nu}\left(p, q^{\prime}, p^{\prime}\right) \\
\Gamma_{D_{17}}^{\mu \nu}=\Gamma_{D_{15}}^{\mu \nu}\left(p^{\prime}, q^{\prime}, p\right) ; \Gamma_{D_{18}}^{\mu \nu}=\Gamma_{D_{15}}^{\mu \nu}\left(p^{\prime}, q, p\right) ; \Gamma_{D_{24}}^{\mu \nu}=\Gamma_{D_{23}}^{\mu \nu}\left(p, q^{\prime}, p^{\prime}\right) ; \Gamma_{D_{25}}^{\mu \nu}=\Gamma_{D_{23}}^{\mu \nu}\left(p^{\prime}, q^{\prime}, p\right) \\
\Gamma_{D_{26}}^{\mu \nu}=\Gamma_{D_{23}}^{\mu \nu}\left(p^{\prime}, q, p\right) ; \Gamma_{D_{28}}^{\mu \nu}=\Gamma_{D_{27}}^{\mu \nu}\left(k, p, q^{\prime}, p^{\prime}\right)
\end{gathered}
$$

Diagrams $\Gamma_{D_{3}}^{\mu \nu} ; \Gamma_{D_{13}}^{\mu \nu} ; \Gamma_{D_{14}}^{\mu \nu} ; \Gamma_{D_{21}}^{\mu \nu}$ and $\Gamma_{D_{22}}^{\mu \nu}$ vanish, so they do not contribute. The calculating allow us to arrange terms of total 4-point vertex function in term of:

$$
\begin{aligned}
\Gamma_{\mu \nu}=C_{0} g_{\mu \nu}+C_{1} k_{\mu} k_{\nu} & +C_{2} k_{\mu} p_{\nu}+C_{3} p_{\mu} k_{\nu}+C_{4} k_{\mu} p_{\nu}^{\prime}+C_{5} p_{\mu}^{\prime} k_{\nu} \\
& +C_{6} p_{\mu} p_{\nu}^{\prime}+C_{7} p_{\mu}^{\prime} p_{\nu}+C_{8} p_{\mu}^{\prime} p_{\nu}^{\prime}+C_{9} p_{\mu} p_{\nu},
\end{aligned}
$$


in which factors $C_{i}$ can be computed according to one-loop diagrams. Now factors of transverse component of 4-point vertex function can be determined based on $C_{i}$ as follows:

$$
\begin{aligned}
B_{11} & =-\frac{k^{2}}{k k^{\prime}} C_{1}+\frac{k^{2}\left(k p+p^{2}-p p^{\prime}\right)}{\left(k k^{\prime}\right)^{2}} C_{2}-\frac{k p}{k k^{\prime}} C_{3}+\frac{k^{2}\left(-p^{2}+p p^{\prime}+k p^{\prime}\right)}{\left(k k^{\prime}\right)^{2}} C_{4} \\
& +\frac{k p\left(-p^{2}+p p^{\prime}+k p^{\prime}\right)}{\left(k k^{\prime}\right)^{2}} C_{6}-\frac{k p^{\prime}}{k k^{\prime}} C_{5}+\frac{k p^{\prime}\left(k p+p^{2}-p p^{\prime}\right)}{\left(k k^{\prime}\right)^{2}} C_{7} \\
& +\frac{k p^{\prime}\left(-p^{\prime 2}+p p^{\prime}+k p^{\prime}\right)}{\left(k k^{\prime}\right)^{2}} C_{8}+\frac{k p\left(k p+p^{2}-p p^{\prime}\right)}{\left(k k^{\prime}\right)^{2}} C_{9} \\
B_{22} & =\frac{1}{4} \frac{C_{6}+C_{7}+C_{8}+C_{9}}{\left(k p^{\prime}\right)\left(-2 k p+k p^{\prime}\right)+(k p)^{2}+k^{2}\left(k^{2}+2 k p-2 k p^{\prime}\right)} \\
B_{23} & =\frac{1}{4} \frac{C_{6}-C_{7}+C_{8}-C_{9}}{\left(k p^{\prime}\right)\left(-2 k p+k p^{\prime}\right)+(k p)^{2}+k^{2}\left(k^{2}+2 k p-2 k p^{\prime}\right)} \\
B_{32} & =\frac{1}{4} \frac{2 C_{2}+2 C_{4}-C_{6}+C_{7}+C_{8}-C_{9}}{\left(k p^{\prime}\right)\left(-2 k p+k p^{\prime}\right)+(k p)^{2}+k^{2}\left(k^{2}+2 k p-2 k p^{\prime}\right)} \\
B_{33} & =-\frac{1}{4} \frac{2 C_{2}-2 C_{4}+C_{6}+C_{7}-C_{8}-C_{9}}{\left(k p^{\prime}\right)\left(-2 k p+k p^{\prime}\right)+(k p)^{2}+k^{2}\left(k^{2}+2 k p-2 k p^{\prime}\right)}
\end{aligned}
$$

\section{CONCLUSION}

Using the Ward-Takahashi identity to represent 3 and 4-point vertex functions as the sum of two parts. First, the longitudinal part of 3-point vertex can be written in terms of complete scalar propagator while for 4-point vertex, this part is presented in terms of scalar propagators and transverse part of 3-point vertex function. Second, transverse parts are not presented in terms of fixed components which depends on specific orders of the perturbative theory. Finally this method can be used to derive transverse part of 3-point and 4-point vertex functions at higher orders of the perturbative theory.

\section{ACKNOWLEDGMENTS}

This research is funded by Vietnam National Foundation for Science and Technology Development (NAFOSTED) under grant number 103.01-2011.63.

\section{REFERENCES}

[1] H. N. Long, Basics of Particle Physics, 2006, Statistic Publisher.

[2] A. Bashir, Y. Concha-Sanchez and R. Delbourgo, Phys. Rev. D76, (2007) 068009.

[3] A. Bashir, Y. Concha-Sanchez and R. Delbourgo, Phys. Rev. D80, (2009) 045007.

[4] P. V. Dong and H. N. Long, The Economical $S U(3) \times S U(2) \times U(1)$ Gauge Model, Publishing House for Natural Science and Technology, Vietnam Academy of Science and Technology, 2008.

Received 31 July 2012. 